\title{
Phase diagram of the bose Hubbard model
}

\author{
J. K. Freericks $(a)$ and H. Monien $(b)$. \\ Institute for Theoretical Physics, University of California, Santa Barbara, CA 93106
}

(July 7,1993$)$

\begin{abstract}
The first reliable analytic calculation of the phase diagram of the bose gas on a $d$-dimensional lattice with on-site repulsion is presented. In one dimension, the analytic calculation is in excellent agreement with the numerical Monte Carlo results. In higher dimensions, the deviations from the Monte Carlo calculations are larger, but the correct shape of the Mott insulator lobes is still obtained. Explicit expressions for the energy of the Mott and the "defect" phase are given in a strong-coupling expansion.
\end{abstract}

Typeset using REVTEX 
Strongly interacting quantum systems continue to challenge theoretical physics. Fermionic examples of strong correlations include high temperature superconductors, heavy fermion materials and metal-insulator transitions. The study of these systems has been confined to either numerical simulations which are plagued by finite-size effects (with the notable exception of the infinite-dimensional expansion) or uncontrollable approximations. Similar problems arise in strongly interacting bosonic systems which have attracted a lot of recent interest [1] [1]. Physical realizations include short correlation length superconductors, granular superconductors, Josephson arrays, and the dynamics of flux lattices in type II superconductors. The relevant physics of these problems is contained in the bose Hubbard Hamiltonian which describes the competition between kinetic energy and potential energy effects. Various aspects of this model were investigated analytically by mean-field theory [1.5], by renormalization group techniques [1,3] and by projection methods [6]. The bose Hubbard model was also studied with Quantum Monte Carlo methods (QMC) by Batrouni et al. [2] in one dimension $(1+1)$ and by Krauth and Trivedi [7] in two dimensions $(2+1)$. In this contribution, we show that the phase diagram obtained from a strong-coupling expansion has the correct dependence on the dimensionality of the spatial lattice, and agrees with the QMC calculations (to within a few percent).

We study the minimal model which contains the key physics of the strongly interacting bose system - the competition between kinetic and potential energy effects:

$$
H=-\sum_{i j} t_{i j} b_{i}^{\dagger} b_{j}-\mu \sum_{i} \hat{n}_{i}+\frac{1}{2} U \sum_{i} \hat{n}_{i}\left(\hat{n}_{i}-1\right) \quad, \quad \hat{n}_{i}=b_{i}^{\dagger} b_{i}
$$

where $b_{i}$ is the boson annihilation operator at site $i, t_{i j}$ is the hopping matrix element between the site $i$ and $j, U$ is the strength of the on-site repulsion, and $\mu$ is the chemical potential. The approximate form of the zero temperature $(T=0)$ phase diagram can be understood by starting from the strong-coupling or "atomic" limit [1,8,8]. In this limit, the kinetic energy vanishes $\left(t_{i j}=0\right)$ and every site is occupied by a fixed number of bosons, $n_{0}$. The ground-state boson occupancy $\left(n_{0}\right)$ is then chosen in such a way as to minimize the on-site energy. If the chemical potential, $\mu=\left(n_{0}+\delta\right) U$, is parametrized in terms of the deviation, 
$\delta$, from integer filling $n_{0}$, then the on-site energy is $E\left(n_{0}\right)=-\delta U n_{0}-\frac{1}{2} U n_{0}\left(n_{0}-1\right)$, and the energy to add a boson onto a particular site satisfies $E\left(n_{0}+1\right)-E\left(n_{0}\right)=-\delta U n_{0}$. Thus for a nonzero $\delta$, a finite amount of energy (gap) is required to move a particle through the lattice. The bosons are localized, producing a Mott insulator. This energy gap decreases with increasing strength of the hopping matrix elements until it vanishes and the bosons condense into the superfluid phase. For $\delta=0$ the energy of the two different boson densities is degenerate $\left[E\left(n_{0}\right)=E\left(n_{0}+1\right)\right]$ and no energy is needed to add or extract a particle; i.e., the compressibility is finite. As the strength of the hopping matrix elements increases, the range of the chemical potential $\mu$ about which the system is incompressible decreases. The Mott-insulator phase will completely disappear at a critical value of the hopping matrix elements.

We are interested in the determination of the phase boundary between the (incompressible) Mott insulator and the (compressible) superfluid phase. A strong-coupling expansion for this boundary is determined by calculating both the energy of the Mott insulating phase and of a defect phase (which contains an extra hole or particle) in a perturbative expansion of the kinetic energy. At the point where the energy of the Mott phase is degenerate with the defect phase, the system becomes compressible, and, since there is no disorder, also superfluid [国]. There are two distinct cases for the defect phase: $\delta<0$ corresponds to adding a boson to the Mott-insulator phase (with $n_{0}$ bosons per site); and $\delta>0$ corresponds to adding a hole to the Mott-insulator phase (with $n_{0}+1$ ) bosons per site. The phase boundary will depend on the number of bosons per site, $n_{0}$, of the initial Mott insulator phase.

To zeroth order in $t / U$ the Mott insulating state is given by

$$
\left|\Psi_{\text {Mott }}\left(n_{0}\right)\right\rangle^{(0)}=\prod_{i=1}^{N} \frac{1}{\sqrt{n_{0} !}}\left(b_{i}^{\dagger}\right)^{n_{0}}|0\rangle
$$

where $n_{0}$ is the number of bosons on each site, $N$ is the number of sites in the lattice and $|0\rangle$ is the vacuum state. The defect phase is characterized by one additional particle (hole) which moves coherently throughout the lattice. To zeroth order in $t / U$ the wave function for the "defect phase" is determined by degenerate perturbation theory: 


$$
\begin{aligned}
\left|\Psi_{\text {Def }}\left(n_{0}\right)\right\rangle_{\text {particle }}^{(0)} & =\frac{1}{\sqrt{n_{0}+1}} \sum_{i} f_{i} b_{i}^{\dagger}\left|\Psi_{\text {Mott }}\left(n_{0}\right)\right\rangle^{(0)} \\
\left|\Psi_{\text {Def }}\left(n_{0}\right)\right\rangle_{\text {hole }}^{(0)} & =\frac{1}{\sqrt{n_{0}}} \sum_{i} f_{i} b_{i}\left|\Psi_{\text {Mott }}\left(n_{0}\right)\right\rangle^{(0)}
\end{aligned}
$$

where the $f_{i}$ is the eigenvector of the hopping matrix $t_{i j}$ with the lowest eigenvalue [10].

For simplicity we will only consider hopping between the nearest neighbors of a hypercubic lattice in $d$-dimensions. The number of nearest neighbors is denoted by $z=2 d$ and the hopping matrix element by $t$; the minimum eigenvalue of the hopping matrix is $-z t$. The many body version of standard Rayleigh-Schrödinger perturbation theory is employed throughout. To third order in $t / U$, the energy of the Mott state with $n_{0}$ bosons per site becomes

$$
E_{\mathrm{Mott}}\left(n_{0}\right)=N\left[-\delta U n_{0}-\frac{1}{2} U n_{0}\left(n_{0}+1\right)-\frac{z t^{2}}{U} n_{0}\left(n_{0}+1\right)\right]
$$

which is proportional to the number of sites. Note that the odd-order terms vanish. The energy difference between the Mott insulating phase and the defect phase with an additional particle $(\delta<0)$ satisfies

$$
\begin{aligned}
E_{\text {Def }}^{(\text {particle })}\left(n_{0}\right)-E_{\text {Mott }}\left(n_{0}\right) & =-\delta^{(\text {particle })} U-z t\left(n_{0}+1\right)+\frac{z t^{2}}{U} \frac{n_{0}\left(5 n_{0}+4\right)}{2}-\frac{z^{2} t^{2}}{U} n_{0}\left(n_{0}+1\right) \\
& +\frac{t^{3}}{U^{2}} n_{0}\left(n_{0}+1\right)\left[\left(-2 z^{3}+\frac{25}{4} z^{2}-4 z\right) n_{0}+\left(-z^{3}+\frac{7}{2} z^{2}-2 z\right)\right]
\end{aligned}
$$

to third order in $t / U$; while the energy difference between the Mott insulating phase and the defect phase with an additional hole $(\delta>0)$ satisfies

$$
\begin{aligned}
E_{\text {Def }}^{(\text {hole })}\left(n_{0}\right)-E_{\mathrm{Mott}}\left(n_{0}\right) & =\delta^{(\text {hole })} U-z t n_{0}+\frac{z t^{2}}{U} \frac{\left(n_{0}+1\right)\left(5 n_{0}+1\right)}{2}-\frac{z^{2} t^{2}}{U} n_{0}\left(n_{0}+1\right) \\
& +\frac{t^{3}}{U^{2}} n_{0}\left(n_{0}+1\right)\left[\left(-2 z^{3}+\frac{25}{4} z^{2}-4 z\right) n_{0}+\left(-z^{3}+\frac{11}{4} z^{2}-2 z\right)\right]
\end{aligned}
$$

These results have been verified by small-cluster calculations on two and four-site clusters. Note that the energy difference in Eqs. (5) and (6) is independent of the lattice size $N$.

The phase boundary between the incompressible Mott phase and the compressible superfluid phase occurs when the energy difference between the two different phases vanishes [11]. The two branches of the Mott phase boundary meet when 


$$
\delta^{(\text {particle })}\left(n_{0}\right)+1=\delta^{(\text {hole })}\left(n_{0}\right) .
$$

The additional one on the left hand side arises because $\delta$ is measured from the point $\mu / U=$ $n_{0}$. Equation (7) may be used to estimate the critical value of the hopping matrix element, $t_{\text {critical }}\left(n_{0}\right)$, beyond which no Mott-insulator phase exists.

In one dimension, the upper boundary of the Mott insulator lobe (with a particle density of $n_{0}$ ) is given by

$$
\delta^{(\text {particle })}\left(n_{0}, t / U\right)=-2\left(n_{0}+1\right)(t / U)+n_{0}^{2}(t / U)^{2}+n_{0}\left(n_{0}+1\right)\left(n_{0}+2\right)(t / U)^{3}
$$

to third order in $t / U$, and the lower boundary is given by

$$
\delta^{(h o l e)}\left(n_{0}, t / U\right)=2 n_{0}(t / U)-\left(n_{0}+1\right)^{2}(t / U)^{2}+n_{0}\left(n_{0}+1\right)\left(n_{0}-1\right)(t / U)^{3} .
$$

The slope of the phase boundaries about the point $\mu=n_{0} U$ are equal in magnitude to first order $\left[\lim _{t \rightarrow 0} \frac{d}{d t} \delta^{\text {particle }}\left(n_{0}, t / U\right)=-\lim _{t \rightarrow 0} \frac{d}{d t} \delta^{h o l e}\left(n_{0}+1, t / U\right)\right]$, but change in magnitude as a function of the density $\left(n_{0}\right)$, implying that the Mott-phase lobes always have an asymmetrical shape.

The strong-coupling expansion for the $t, \mu$ phase diagram in one dimension is compared to the QMC results of Batrouni et al. [2] in Figure 1. The solid lines indicate the phase boundary between the Mott-insulator phase and the superfluid phase at zero temperature as calculated from Eq. (8) and Eq. (9). The squares are the results of the QMC calculation at a small but finite temperature $T=U / 2$ [2]. Note that the overall agreement of the two calculations is excellent. For example, the critical value of the chemical potential for the first lobe $\left(n_{0}=1\right)$ satisfies $\delta_{\text {critical }} \approx-0.755$ so that the critical value of the hopping matrix element is $(t / U)_{\text {critical }}=0.215$, while the QMC calculations found $(t / U)_{\text {critical }}=0.215 \pm 0.01$ [2]. A closer examination shows that the first lobe $\left(n_{0}=1\right)$ has a systematic deviation at larger values of $t$. This is most likely a finite-temperature effect, since the Mott-insulator phase becomes more stable at higher temperatures [5].

It is known from the scaling theory of Fisher et al. [1] that the phase transition at the tip of the Mott lobe is in the universality class of the $(d+1)$ dimensional $X Y$ model. Although a 
finite-order perturbation theory cannot describe the physics of the tricritical point correctly, it turns out that the density fluctuations dominate the physics of the phase transition even close to the tricritical point. Note how the Mott lobes have a cusp-like structure in one dimension, mimicking the Kosterlitz-Thouless behavior of the critical point.

Figure 2 presents the strong-coupling expansion for the $t, \mu$ phase diagram in two dimensions. For comparison, the tricritical point of the first Mott-insulator lobe as obtained by the QMC simulations of Krauth and Trivedi [7] is marked with a solid square. Their numerical calculation gives a critical value of $(t / U)_{\text {critical }}=0.122 \pm 0.01$, whereas our calculation yields $(t / U)_{\text {critical }} \approx 0.136$ which is in reasonable agreement. As already mentioned above we cannot hope to describe the physics close to tricritical point with our approach, but note that the qualitative shape of the Mott lobes has changed from one dimension to two dimensions, mimicking the "smooth" critical behavior of the $X Y$ model in three or larger dimensions.

Finally the strong-coupling expansion is compared to the exact calculation in infinite dimensions [1]. In infinite dimensions, the hopping matrix element must scale inversely with the dimension [12], $t=t^{*} / d, t^{*}=$ finite, producing the mean field theory of Ref. [1]. In Figure 3 the strong-coupling expansion (solid line) is compared to the exact solution (dashed line). Even in infinite dimensions, the agreement of the strong-coupling expansion with the exact results is quite good.

We have repeatedly compared a strong-coupling expansion to the numerical QMC simulations for the incompressible-compressible phase boundary of the bose Hubbard model. A mean-field treatment of the bose Hubbard model (e.g. [1,5]) cannot capture the physics of the one dimensional system which is completely dominated by fluctuations. The dimensionality only enters as a trivial prefactor in integrals over the phase space. For this reason, mean-field theories will always give a concave shape to the Mott-insulator lobes independent of the dimension. A strong-coupling expansion, on the other hand, easily distinguishes the shape difference from one dimension to higher dimensions and shows that a proper treatment of density fluctuations is critical in determining the Mott-insulator to superfluid transition. 
In conclusion we have described an analytical method to accurately calculate the phase diagram of the bose Hubbard model in any dimension. Extensions of these techniques to include disorder will be presented separately.

\section{ACKNOWLEDGMENTS}

We would like to thank R. Scalettar, G. Batrouni and K. Singh, for providing us with the Quantum Monte Carlo data for the one dimensional bose Hubbard model and for many useful discussions. This research was supported in part by the NSF under Grant No. PHY89-04035 and DMR90-02492. 


\section{REFERENCES}

(a) Address from September 1. 1993 on: Physics Department Davis, University of California, Davis, California 95616.

(b) Address from September 1. 1993 on: Theoretische Physik, ETH Höngerberg, CH-8093 Zürich, Switzerland.

[1] M.P.A. Fisher, P.B. Weichman, G. Grinstein, and D.S. Fisher, Phys. Rev. B40, 546 (1989).

[2] R.T. Scalettar, G.G. Batrouni, and G.T. Zimanyi, Phys. Rev. Lett. 66, 3144 (1991), P. Niyaz, R.T. Scalettar, C.Y. Fong and G.G Batrouni, Phys. Rev. B44, 7143 (1991). G.G. Batrouni and R.T. Scalettar, Phys. Rev. B46, 9051 (1992);

[3] D.S. Rokhsar and B.G. Kotliar, Phys. Rev. B 44, 10328 (1991); K. G. Singh and D. S. Rokhsar, Phys. Rev. B46, 3002 (1992).

[4] E.S. Sörensen, M. Wallin, S.M. Girvin, A.P. Young, Phys. Rev. Lett. 69, 828 (1992).

[5] A.P. Kampf and G.T. Zimanyi, Phys. Rev. B47, 279 (1993).

[6] W. Krauth, M. Caffarel, and J.P. Bouchaud, Phys. Rev. B45, 3137 (1992); K. Sheshadri, H. R. Krishnamurty, R. Pandit and T. V. Ramkrishnan, Europhys. Lett. 22, 257 (1993).

[7] W. Krauth and N. Trivedi, Europhysics Lett. 14, 627 (1991); W. Krauth, N. Trivedi, and D. Ceperley, Phys. Rev. Lett. 67, 2307 (1991).

[8] T. Giamarchi and H. J. Schulz, Phys. Rev. B37, 325 (1988).

[9] M. Ma, B. I. Halperin, and P. A. Lee, Phys. Rev. B34, 3136 (1986).

[10] The hopping matrix is assumed to have a nondegenerate lowest eigenvalue.

[11] The compressibility is assumed to vanish continuously at the phase boundary.

[12] E. Müller-Hartmann, Int. J. of Mod. Phys. B 3, 2169 (1989). 


\section{FIGURES}

FIG. 1. The $t, \mu$ phase diagram of the bose Hubbard model in one dimension $(d=1)$. The solid lines give the phase boundaries of the Mott insulator to the superfluid state as determined from a third-order strong-coupling calculation. The squares are the result of the QMC calculation of Batrouni et al. [2].

FIG. 2. The $t, \mu$ phase diagram of the bose Hubbard model in two dimensions $(d=2)$. The solid lines give the phase boundaries of the Mott insulator to the superfluid state as determined from a third-order strong-coupling calculation. The point indicates the tricritical point as determined by the QMC calculation of Krauth and Trivedi [7].

FIG. 3. The $t, \mu$ phase diagram of the bose Hubbard model in infinite dimensions $(d \rightarrow \infty)$. The solid lines give the phase boundaries of the Mott insulator to the superfluid state as determined from a third-order strong-coupling calculation. The dashed lines are the result of the mean field calculation of Fisher et al. [1]. 